\title{
Fractionation in radiotherapy: results of a Canadian randomised clinical trial
}

\author{
J. Yarnold \\ Royal Marsden Hospital, London, UK.
}

\begin{abstract}
Historical assumptions concerning the optimal fractionation schedules for women with breast cancer are being challenged by the early results of randomised clinical trials. Multiple small fractions of 2.0 Gy or less are optimal for squamous cell carcinomas, which are clearly less sensitive to fraction size than the surrounding dose-limiting normal tissues. Breast cancer may be different in showing comparable sensitivity to fraction size as the healthy tissues of the breast and underlying ribcage. If this is confirmed, it means that fewer, larger fractions confer the same benefit as standard 2.0 Gy schedules, provided appropriate downward corrections are made to the total dose. The approach also lends itself to tests of acceleration, shorter treatment times being of obvious interest to patients and possibly of therapeutic benefit in their own right.
\end{abstract}

Keywords: Breast cancer; Fractionation; Normal tissues; Radiotherapy

Most people assume that human tumour responses are relatively insensitive to fraction size compared to dose-limiting late reacting normal tissues. This assumption is consistent with the responses of animal tumours and of human squamous carcinomas of the cervix uteri, lung and head and neck [1-3]. These data show that as fraction size increases above 2.0 Gy, the ratio of tumours cured to late adverse effects falls, a highly unfavourable relationship. Even though, this may not apply to all tumour types. The fractionation sensitivity of normal tissues and tumours is well described by a linear-quadratic model [4]. The $\alpha / \beta$ value in this simple empirical formula (units Gy) is a practical descriptor of the sensitivity to fraction size. Values of $\alpha / \beta$ in the range 1-6 Gy are typical of late responding tissues, with higher values ( $\geqslant 10 \mathrm{~Gy}$ ) typical of squamous carcinomas and early responding normal tissues. The hypothesis relevant to the present discussion is that $\alpha / \beta$ values for breast cancer are closer to those of late normal tissue responses

Correspondence to: J. Yarnold, Academic Radiotherapy Department, The Royal Marsden Hospital, Downs Road, Sutton, Surrey SM2 5PT, UK E-mail: John.Yarnold@icr.ac.uk

Publication date 30/06/05 BCO/371/2005/FO than to human squamous carcinoma. The implication of this hypothesis is that hypofractionation (fraction sizes $>2$ Gy) should be evaluated for the treatment of primary breast cancer (the following arguments do not apply to treatment of the lymphatic pathways due to the very high fractionation sensitivity of the brachial plexus).

An $\alpha / \beta$ value in the range $4-5$ Gy was first estimated for the response of locally advanced and recurrent chest wall breast cancer in the early 1950s, and analysed using the linear quadratic model in the mid-1980s [5,6]. More recently, a direct estimate of $4.1 \mathrm{~Gy}(95 \% \mathrm{Cl} 1.0-9.7)$ was reported for the fractionation sensitivity of breast cancer in the Royal Marsden Hospital/Gloucestershire Oncology Centre Breast Fractionation Trial $(N=1410)$ [7]. Meanwhile, a randomised comparison of $50 \mathrm{~Gy}$ in 25 fractions of 2.0 and 42.5 Gy in 16 fractions of $2.67 \mathrm{~Gy}(N=1234)$ in Ontario reported no significant differences in local tumour recurrence between arms [8]. If the two Ontario schedules are truly iso-effective with respect to tumour control, it implies an $\alpha / \beta$ value of $3 \mathrm{~Gy}$ (higher if tumour repopulation is relevant - see below).

The UK Standardisation of Radiotherapy (START) Trial has also tested the fractionation sensitivity of breast cancer in a design that incorporates two 
randomisations: Trial $\mathrm{A}(N=2236)$ is an explanatory trial that tests $50 \mathrm{~Gy}$ in 25 fractions of 2.0 Gy against two dose levels of a 13-fraction regimen delivering 3.0 Gy or 3.2 Gy fractions over 5 weeks; Trial B $(N=2215)$ is a pragmatic trial testing 50 Gy in 25 fractions against $40 \mathrm{~Gy}$ in 15 fractions of $2.67 \mathrm{~Gy}$. If the relatively high fractionation sensitivity of breast cancer is confirmed (no results have been reported to date), the implications are that larger fraction sizes have no disadvantages, and perhaps significant advantages, for women with primary breast cancer. Since it is unlikely that 13 - or 15 -fraction schedules would represent the limit of what might be achieved, further studies are justified to test the limits of hypofractionation in breast cancer.

Hypofractionation lends itself to acceleration, taking advantage of the relative sparing of early skin reactions as fraction size increases and the absence of a significant time dependency for late adverse effects. Tumour repopulation has recently been tested as a determinant of treatment outcome in the context of adjuvant systemic therapy. In a randomised comparison of conventional 3-weekly schedules of doxorubicin and cyclophosphamide with the same chemotherapy doses given at 2-weekly intervals (using growth factors to accelerate marrow recovery), the hazard ratios for disease-free and overall survival associated with 2-weekly chemotherapy were 0.74 $(P=0.01)$ and $0.69(P=0.01)$, respectively in 2005 patients [9]. Recent reports of accelerated radiotherapy fractionation in head and neck cancer indicate that very modest shortening of treatment has a detectable impact on local control. In a trial of 1476 patients randomised to 5 (control arm) or 6 (test arm) fractions per week of conventional radiotherapy, local tumour control in the test arm was $76 \%$ patients compared to $64 \%$ in the control group [10]. In this study, shortening treatment by only 7 days was associated with a $12 \%$ absolute reduction in local recurrence at the primary site, a reduction in the odds of recurrence of $16 \%$. The possible implications for primary breast cancer are that modest reductions in treatment time, along the lines of the 3 weeks tested in the Canadian trial, may translate into worthwhile gains in tumour control.

Finally, the implications of advanced radiotherapy techniques for delivering the biological advantages of hypofractionation are worth considering. Rather than increase dose intensity by increasing the number of 2.0 Gy fractions, it creates opportunities for escalating dose intensity by modulating fraction size (this argument does not hold for the lymphatic pathways). Even if the fractionation sensitivity of breast cancer is not quite as great as the normal tissues of the breast, shorter overall treatment times needed to deliver concomitant boost using intensity modulated radiotherapy could be advantageous if tumour proliferation is a significant determinant of local control. The implications of dose escalated intensity modulated radiotherapy are under test in the proposed UK IMPORT Trial. The hypothesis is that higher doses per fraction to high-risk areas and lower fraction sizes to low-risk areas of the breast will offer a clinically superior and cost-effective approach of matching dose intensity to tumour recurrence risk compared to standard sequential boost techniques. In conclusion, future prospects for exploiting the biology of hypofractionation in breast cancer using advanced radiotherapy technologies look bright, with prospects for testing the limits of accelerated hypofractionation and dose escalated intensity modulated radiotherapy by the end of the decade.

\section{References}

1. Yarnold JR, Owen JR, et al. Fraction sensitivity of change in breast appearance after radiotherapy for early breast cancer: long-term results of a randomised trial. Radiother Oncol 2002; 64: S25.

2. Thames HD, Hendry JH, Moore JV, et al. The high steepness of dose-response curves for late-responding normal tissues. Radiother Oncol 1989; 15(1): 49-53.

3. Thames HD, Bentzen SM, Turesson I, et al. Time-dose factors in radiotherapy: a review of the human data. Radiother Oncol 1990; 19: 219-235.

4. Jones B, Dale RG, Deehan C, et al. The role of biologically effective dose (BED) in clinical oncology. Clin Oncol (R Coll Radiol) 2001; 13: 71-81.

5. Douglas G. Superfractionation: its rationale and anticipated benefits. Int J Radiat Oncol Biol Phys 1982; 8: 1143-1153.

6. Cohen L. Radiotherapy in breast cancer I. The dose-time relationship theoretical considerations. Br J Radiol 1952; 25: 636-642.

7. Yarnold J, Ashton A, Bliss J, Homewood J, Harper C, Hanson J, Haviland J, Bentzen S, Owen R. Fractionation sensitivity and dose response of late adverse effects in the breast after radiotherapy for early breast cancer: long-term results of a randomised trial. Radiother Oncol 2005; 75(1): 9-17.

8. Whelan T, MacKenzie R, Julian J, et al. Randomized trial of breast irradiation schedules after lumpectomy for women with lymph node-negative breast cancer. $J$ Natl Cancer Inst 2002; 94: 1143-1150.

9. Citron ML, Berry DA, Cirrincione C, et al. Randomized trial of dose-dense versus conventionally scheduled and sequential versus concurrent combination chemotherapy as postoperative adjuvant treatment of node-positive primary breast cancer: first report of Intergroup Trial C9741/Cancer and Leukemia Group B Trial 9741. J Clin Oncol 2003; 21: 1431-1439.

10. Overgaard J, Hansen HS, Specht L, et al. Five compared with six fractions per week of conventional radiotherapy of squamous-cell carcinoma of head and neck: DAHANCA 6 and 7 randomised controlled trial. Lancet 2003; 362 933-940. 